\title{
Stepped Strabismus Surgery
}

\author{
Amr Elkamshoushy (10 \\ Ahmed Kassem (iD) \\ Department of Ophthalmology, Faculty of \\ Medicine, University of Alexandria, \\ Alexandria, Egypt
}

Purpose: To present stepped strabismus surgery as a novel technique in cases of small to moderate angle strabismus.

Methods: Retrospective chart review of cases of stepped strabismus surgery from 2010 untill 2018. In stepped surgery, the first muscle is operated on under rapid induction-recovery IV propofol infusion. Patient is assessed in the OR. If deemed necessary, other muscles are operated on under general anesthesia. No adjustable sutures are used.

Results: The technique was used in 22 cases of superior oblique palsy (SOP) (primary position hypertropia in the range of 12-25 prism diopters) and 29 horizontal strabismus cases (angles in the range 12-20 prism diopters). The first step was an inferior oblique myectomy in the SOP cases and a single rectus recession in the horizontal cases. After intraoperative assessment, 31\% (16/51) needed additional muscle surgery. After 6 months of follow up, the overall reoperation rate was $9 \%$. The technique was well tolerated by all patients.

Conclusion: Stepped strabismus surgery is a useful technique for small to moderate angle strabismus cases with the potential for reducing the number of extraocular muscles operated on without compromising the surgical outcome.

Keywords: anesthesia, anesthetics, intravenous, oculomotor muscles/surgery, propofol, retrospective studies, strabismus/surgery

\section{Introduction}

Management options of adult patients with vertical or horizontal strabismus include fixed suture surgery or adjustable suture surgery with early or delayed adjustment depending on the surgeon's preference. In small to moderate angles the decision whether to operate on one muscle or more is not always easy. Overcorrection is a feared outcome in cases where two muscles are operated on. Operating on one muscle may cause undercorrection requiring reoperation or in cases of adjustable surgery requiring too much adjustment with unwanted incomitance. ${ }^{1-3}$ Alternative techniques such as partial tenotomy and mini-plication were devised to address small-angle strabismus with the limitation of correcting only up to 10 prism diopters (PD) in the case of unilateral surgery. ${ }^{4-6}$ Similarly, the decision of whether to operate on one or two muscles in cases of superior oblique palsy is not always clear. Conventional teaching recommends using the inferior oblique weakening procedure solely for vertical deviations less than $15 \mathrm{PD}$ in primary position. ${ }^{7,8}$ Emerging evidence suggests that inferior oblique myectomy can be used as a single procedure for vertical deviations up to $30 \mathrm{PD} .^{9}$

Stepped strabismus surgery is a novel method that is useful for cases of small to moderate angle horizontal and vertical strabismus. It obviates the need for adjustable sutures and allows for intraoperative optional increments on fixed suture 
single muscle surgery. This technique allows the intraoperative detection of large undercorrections that may occur with single muscle surgery and hence adding more muscle surgery. The purpose of this article is to describe this new technique and present its results and safety for cases of small to moderate angle strabismus.

\section{Subjects and Methods}

Approval for the study was obtained from the University of Alexandria institutional review board before commencing the study. The study adhered to the tenets of the Declaration of Helsinki. Patient consent to review their medical records was not required by the University of Alexandria Institutional Review Board and this was waived due to the retrospective nature of the study. Directly identifying information (e.g. names, addresses) were safeguarded and maintained under controlled conditions.

Medical records of all patients who underwent stepped strabismus surgery between January 2010 and January 2019 were retrospectively reviewed. Files were only included if they had a minimum follow up of 6 months with full measurements recorded. Cases of horizontal and vertical strabismus were included. All patients were operated on by the same surgeon (Amr Elkamshoushy).

Preoperative assessment included full ophthalmological examination, and angle of strabismus measurement was measured using alternate prism and cover test. Krimsky test was used in cases where vision in one eye did not allow the use of prism and cover. The pre and postoperative angle of strabismus was noted and statistically analyzed. The success rate, defined as alignment within 8 prism diopters of orthotropia in horizontal cases and within 4 prism diopters of orthotropia in vertical cases measured at the last follow up was tabulated. The number of re-operations was reported as well. Differences in outcome were stratified for patients with esotropia, exotropia and hypertropia.

\section{Surgical Technique}

The method of stepped surgery is doing a first step under general anesthesia (GA), followed by rapid recovery in the operating room (OR) to assess the immediate results after recovery. If needed, a second step is done under GA. No adjustable sutures are used. The first step is always a one muscle surgery. Shorter anesthesia duration allows faster recovery. To help reduce the time of anesthesia, the surgical plan is reviewed and the surgeon scrubs before the patient is admitted to the OR. Once admitted, the patient is attached to monitors for $\mathrm{ECG}, \mathrm{SpO}_{2}$ and non-invasive blood pressure. Oxygen is administered using nasal cannula with flow $2 \mathrm{~L} /$ minute. Anesthesia is started using a small dose of fentanyl $0.5 \mu \mathrm{g} / \mathrm{kg}$ while the patient is being prepped and draped then a loading dose of propofol $0.5 \mathrm{mg} / \mathrm{kg}$ is injected. ${ }^{4,10}$ Surgery commences immediately after the induction. Supplemental dose of propofol is used by continuous infusion at a rate of $80 \mu \mathrm{g} / \mathrm{kg} / \mathrm{min}$ using a syringe pump. For all esotropia cases, $6 \mathrm{~mm}$ recession of a single medial rectus muscle was performed. Seven $\mathrm{mm}$ recession of a single lateral rectus muscle was the initial procedure in all exotropes. All patients with superior oblique palsy had undergone inferior oblique myectomy as an initial procedure.

At the end of surgical procedure propofol infusion is stopped and the patient is left to recover. The patient is considered fully recovered and ready for angle assessment when they can sit upright unassisted, fix on a target with normal pursuit and saccades and can engage in conversation. Once fully recovered, angle measurement is done in the OR, and if more surgery is deemed necessary, GA is given again using propofol for induction and sevoflurane inhalation for maintenance and a second step is done. The cut-off point for the second step and the details of the second step are shown in Table 1.

\section{Statistical Analysis}

Data were collected and entered to the computer using SPSS (Statistical Package for Social Science) program for statistical analysis (Version 21.0, IBM Corp., Armonk, NY). Data were entered as numerical or categorical, as appropriate. If a significance in the distribution of variables was demonstrated by the Kolmogorov-Smirnov test, non-parametric statistics were used. Data were described using minimum, maximum, mean, standard deviation and $95 \%$ confidence interval of the mean, median and interquartile range. Categorical variables were described using frequency and percentage. Comparisons were carried out between two studied independent notnormally distributed subgroups using Mann-Whitney $U$-test. Chi-square test was used to test association between qualitative variables. Monte Carlo corrections were carried out when indicated.

\section{Results}

A total of 29 patients in the horizontal strabismus group and 22 patients in the superior oblique palsy group were included. Fourteen $(48 \%)$ of the patients in the horizontal strabismus 
Table I Criteria for Intraoperative Second Step

\begin{tabular}{|c|c|c|}
\hline Type of Strabismus & Intraop Assessment After First Step & Second Step \\
\hline Esotropia & $\begin{array}{l}X T=10 \text { to } E T=8 \\
E T 9 \text { to } 15 \\
E T>15\end{array}$ & $\begin{array}{l}\text { No second step } \\
\text { Contralateral MR recession } 4 \\
\text { Contralateral MR recession } 5\end{array}$ \\
\hline Exotropia & $\begin{array}{l}E T=15 \text { to } X T=6 \\
X T 7 \text { to } 15 \\
X T>15\end{array}$ & $\begin{array}{l}\text { No second step } \\
\text { Contralateral LR recession } 5 \\
\text { Contralateral LR recession } 6\end{array}$ \\
\hline Superior oblique palsy & $\begin{array}{l}\mathrm{HT} \pm 4 \\
\mathrm{HT} 5-10 \\
\mathrm{HT}>10\end{array}$ & $\begin{array}{l}\text { No second step } \\
\text { Contralateral IR recession } 3 \text { or Ipsilateral SR recession } 5^{*} \\
\text { Contralateral IR recession } 4 \text { Or Ipsilateral SR recession } 6 *\end{array}$ \\
\hline
\end{tabular}

Note: *lpsilateral SR recession was done in cases with ipsilateral limited depression in adduction (giving the appearance of pseudo overcorrection of the contralateral superior oblique).

group and $10(45 \%)$ patients of the vertical group were males. The mean age $\pm \mathrm{SD}$ of esotropes, exotropes and patients with superior oblique palsy were $27.33 \pm 8.27,30.36$ \pm 9.22 and $29.95 \pm 8.53$, respectively. $8 / 11$ (72\%) of the exotropia group were of the intermittent exotropia type. Demographic data of both groups are summarized in Table 2. The technique was well tolerated for all patients. Recovery time for full assessment ranged between 15-45 minutes for all patients with more than $90 \%$ achieving recovery before 30 minutes. Intraoperative assessment was possible in all patients. None of the patients reported significant adverse events such as nausea, vomiting or vasovagal response. Under-correction requiring an intraoperative second step occurred in 16 out of the 51 patients in the studied group (31.4\%). Six (33.3\%) esotropes, 5 (45.5\%) exotropes and 5 $(22.7 \%)$ patients with superior oblique palsy required a second stage.

There was no statistically significant difference between the three groups in second step rate $\left(\chi^{2}=1.809\right.$, $\mathrm{p}=0.396$ ). Within a year of follow up, 5 of the 51 patients underwent another surgery for unsatisfactory results (9.8\%). Again, no difference in reoperation rate was found between the groups $\left(\chi^{2}=0.054, p=1.00\right)$. Although some patients fell outside the success criteria defined by this study, they were satisfied by the achieved result and did not request a reoperation. Overall success rate as

Table 2 Demographic Data

\begin{tabular}{|c|c|c|c|c|}
\hline & & Esotropia & Exotropia & Superior Oblique Palsy \\
\hline \multirow[t]{4}{*}{ Gender } & & & & \\
\hline & Male & $8 / 18(44 \%)$ & $6 / 11(55 \%)$ & 10/22 (45\%) \\
\hline & Test of significance & \multicolumn{3}{|c|}{$\chi_{(\mathrm{df}=2)}^{2}=0.320$} \\
\hline & $\mathrm{p}$-value & \multicolumn{3}{|c|}{$P_{(M C)}=0.874 \mathrm{NS}$} \\
\hline \multirow[t]{7}{*}{ Age } & & & & \\
\hline & Range & $17-42$ & $19-49$ & $18-45$ \\
\hline & Mean and SD & $27.33 \pm 8.27$ & $30.36 \pm 9.22$ & $29.95 \pm 8.53$ \\
\hline & $\mathrm{KS}$ test of normality & $D=0.146$ & $D=0.195$ & $D=0.1 I I$ \\
\hline & & $\mathrm{P}=0.200 \mathrm{NS}$ & $\mathrm{p}=0.200 \mathrm{NS}$ & $\mathrm{p}=0.200 \mathrm{NS}$ \\
\hline & Test of significance & \multicolumn{3}{|c|}{$Z_{(\mathrm{KW})}=1.317$} \\
\hline & $\mathrm{p}$-value & \multicolumn{3}{|c|}{$p=0.518 \mathrm{NS}$} \\
\hline
\end{tabular}

Abbreviations: $\mathrm{n}$, number of patients; $\chi^{2}$, Chi square test; df, degree of freedom; KS, Kolmogorov-Smirnov; KW, Kruskal-Wallis test; MC, Monte Carlo correction for Pearson Chi-square; NS, statistically not significant $(p \geq 0.05)$. 
Table 3 Preoperative Angle and Postoperative Results

\begin{tabular}{|c|c|c|c|c|}
\hline & & Esotropia & Exotropia & $\begin{array}{l}\text { Superior Oblique } \\
\text { Palsy }\end{array}$ \\
\hline \multicolumn{5}{|l|}{ Preoperative angle (PD) } \\
\hline & $\begin{array}{l}\text { Mean \& } \\
\text { SD }\end{array}$ & $15.9 \pm 3.4$ & $18.0 \pm 2.7$ & $19.4 \pm 4.2$ \\
\hline & Range & 10 to 20 & 12 to 20 & 14 to 30 \\
\hline \multicolumn{5}{|l|}{ Final postoperative angle (PD)* } \\
\hline & $\begin{array}{l}\text { Mean \& } \\
\text { SD }\end{array}$ & $2.9 \pm 5.3$ & $2.4 \pm 6.8$ & $1.6 \pm 3.3$ \\
\hline & Range & -10 to 15 & -12 to 14 & -4 to 8 \\
\hline Intraoperative second step needed & & $6 / 18(33.3 \%)$ & $\begin{array}{l}5 / 11 \\
(45.5 \%)\end{array}$ & $5 / 22(22.7 \%)$ \\
\hline Re-operation** & & $2 / 18(11.1 \%)$ & $\mathrm{I} / \mathrm{II}(9.1 \%)$ & $2 / 22(9.1 \%)$ \\
\hline Re-operation due to undercorrection & & $2 / 2$ & $\mathrm{I} / \mathrm{I}$ & $1 / 2$ \\
\hline Success $* * *$ & & $\begin{array}{l}15 / 18 \\
(83.3 \%)\end{array}$ & $\begin{array}{l}8 / 11 \\
(72.7 \%)\end{array}$ & $18 / 22(81.9 \%)$ \\
\hline $\begin{array}{l}\text { Final success at last follow up not met for patients not needing a second } \\
\text { stage }\end{array}$ & & $2 / 12(11.7 \%)$ & $1 / 6(16.7)$ & $2 / 17(11.7 \%)$ \\
\hline Final success at last follow up not met for patients needing a second stage & & $0 / 6(0 \%)$ & $2 / 5(40 \%)$ & $2 / 5(40 \%)$ \\
\hline
\end{tabular}

Notes: Reported angles are primary position deviations. *Negative values indicate overcorrection angles. **A second surgery was done within a year for unsatisfactory results. ***Defined as primary position deviation within 8 PD in horizontal cases and less than 4 PD in vertical cases after the primary procedure not including further reoperations.

defined in methods was $80.4 \%$ (41 out of 51 cases) with no significant difference between the three groups $\left(\chi^{2}=0.537\right.$, $\mathrm{p}=0.814$ ). Table 3 shows the pre-and postoperative results of the three groups. Failure due to under-correction occurred in 2 out of 3 in each of the esotropia and the exotropia groups (66\%) and in all 4 patients of the superior oblique palsy group not meeting the success criteria.

There was a significant positive correlation between the preoperative deviation and the need for a second step using Pearson correlation test with correlation estimation of 0.5 with $95 \%$ CI $[0.3,1], 0.67$ with $95 \%$ CI $[0.4,1], 0.57$ with $95 \%$ CI $[0.2,1]$ and 0.54 with $95 \% \mathrm{CI}[0.02,1]$ for the entire cohort, superior oblique palsy group, esotropia group and exotropia group, respectively. There was no correlation between the size of the preoperative deviation and failure defined as final postoperative angle not achieved as detailed in the Methods.

\section{Discussion}

This study is the first to present stepped strabismus surgery. This technique is of special use for cases of small to moderate angle strabismus. The success rates of stepped strabismus surgery in esotropia and exotropia compared favorably with those of adjustable suture surgery which ranged between $77.7-91.7 \%$, with the added benefit of potentially reduced operative time and postoperative care unit stay, particularly when a second stage is not required and additionally by eliminating the time needed for the patient to be evaluated and undergo suture adjustment in the postoperative care unit when adjustable sutures are used. ${ }^{11-14}$

Similarly, the re-operation rate in our horizontal group was similar to re-operation rates following adjustable and non-adjustable strabismus surgeries in adults reported by Leffler et al. upon review of a large national private insurance database. ${ }^{15}$

Another advantage of stepped surgery over adjustable is the potential for reducing the number of extraocular muscles operated on without compromising the results. A second muscle is operated on only if needed. In some adjustable cases the recession is completely reversed 
during adjustment by advancing the muscle back to the insertion. Intraoperative assessment after the first muscle in stepped surgery can spare the patient the second muscle recession that would have been reversed in adjustment.

Despite the common conception that inferior oblique myectomy alone cannot fully correct vertical deviations of more than 15 PD, the majority of our patients who had a median hypertropia of 19 PD did not require a second stage following inferior oblique myectomy with an overall excellent outcome at final follow up. Our finding agrees with Akbari et al. who reported successful alignment in $85 \%$ of patients with hypertropia between 15-30 PD in the setting of superior oblique palsy. ${ }^{9}$ The ability to perform an optional second stage procedure could make adopting inferior oblique myectomy as a single procedure for vertical deviations up to $30 \mathrm{PD}$ more acceptable. It is not clear why a second stage was needed the most in the exotropia group.

An important prerequisite to the success of our procedure is allowing smooth emergence from the first anesthetic to permit reliable evaluation in the immediate postoperative period. We were able to successfully achieve good cooperation for examination using the anesthesia protocol described above in almost all patients within 15-30 minutes of termination of the procedure. Rapid induction-recovery anesthesia and intraoperative adjustment has been described by many authors. Ward et al. reported on anesthetic protocol consisting of infusion of mivacurium, alfentanil and propofol and nitrous oxide inhalational anesthesia that was followed by immediate postoperative assessment and adjustment on the operative table whenever indicated. Only one patient of 29 patients could not be reliably assessed in the immediate postoperative period using this technique. ${ }^{10}$ Tatham and Amaya reported similar success using $1 \%$ propofol with $20 \mu \mathrm{g}$ / $\mathrm{mL}$ remifentanil administered via a Diprifusor syringe driver with all patients achieving full recovery within 10 minutes following surgery and able to undergo assessment of alignment. ${ }^{16,17}$ Biglan et al. used a combination of topical proparacaine, subconjunctival mepivacaine injection into the incision site and around the rectus muscle and intravenous sedation using propofol, midazolam and fentanyl to perform intraoperative adjustment in 25 patients. Two of their patients had to be excluded because of discomfort during the procedure and were converted to retrobulbar anesthesia. ${ }^{18}$ Cogen et al. utilized a combination of propofol, midazolam, fentanyl and nitrous oxide to allow immediate postoperative adjustment in 16 patients.
Flumazenil was utilized to quickly reverse the sedative effects of midazolam and $100 \%$ of patients were able to cooperate for motility assessment within 15 minutes of laryngeal mask airway (LMA) removal. ${ }^{19}$ Sharma et al. utilized intravenous sedation with midazolam and fentanyl to perform single-stage adjustable strabismus surgery where patients were adjusted on the operating table at the end of the procedure using a fixation target on the ceiling. ${ }^{20}$ Propofol was the common anesthetic used in most of the described techniques due to its rapid induction and full recovery after cessation of inhalation. It was the main anesthetic we used in our cases. Propofol-only infusion was sufficient to perform horizontal rectus recessions and inferior oblique myectomy without the need for subconjunctival lidocaine.

Unreliability of the assessment of alignment in the immediate postoperative period constitutes a possible limitation to this technique. This issue was raised by Bleik and Karam who reported a mean drift of $7.2 \pm 4.3 \mathrm{PD}$ after 24 hours compared with measurements taken immediately after recovery despite using total intravenous anesthesia. They reported initial under-correction in the case of recessions and initial over-corrections in the case of isolated resections in the immediate postoperative period compared with 24 hours later. ${ }^{21}$ Although our first postoperative measurement of angle was at 1 month postoperative, we did not encounter large unexpected over-corrections or under-corrections in our cohort of patients.

A possible criticism to our technique is the unwanted risk of a second anesthetic episode. This could be outweighed by the fact that a second stage is necessary only if further surgery is needed; $70 \%$ of cases in our cohort did not need a second step. Stepped surgery has the potential of reducing the re-operation rate. This can spare the patient the risk of a second anesthesia and the added psychological stress of another procedure. Stepped surgery with a $30 \%$ potential of another anesthesia in the same procedure may be less stressful than another anesthesia in a reoperation a few months after the first surgery. It should also be noted that our anesthesia protocol involves patients breathing spontaneously thus avoiding the hemodynamic stress associated with intubation. This gives an additional layer of safety. It should be noted that that an additional potential disadvantage is the need to keep the patient in the operating room for additional time while waiting to assess for the need for a second step. This time was used by the surgeon to complete a second case but the cost of additional OR time should be considered. 
Possible limitation of this technique is that only brief procedures can be performed using our anesthesia protocol in the first step. Additionally, since the first procedure is non-adjustable, the option of undoing an over-correction could prove rather difficult. Nevertheless, we did not encounter an immediate over-correction, possibly because of the surgical dose utilized in comparison to the size of the deviation. Another limitation is inability to modify the surgical dose further after performing the second stage unless adjustable sutures are used in the second stage. Additionally, the number of patients in each group was relatively small. Despite these limitations, we believe that stepped strabismus surgery constitutes an important addition to the ever-increasing techniques available at the disposal of the strabismus surgeon.

\section{Acknowledgments}

This work was presented in part at the 45th Annual Meeting of the American Association for Pediatric Ophthalmology and Strabismus, San Diego, CA, March 27-31, 2019. This paper was presented at the Annual Meeting of the American Association for Pediatric Ophthalmology and Strabismus as a poster presentation with interim findings. The poster's abstract was published in "Poster Abstracts" in the Journal of American Association of Pediatric Ophthalmology and Strabismus: https://doi.org/10.1016/j.jaapos.2019.08.019. Institution at which the study was conducted: Department of Ophthalmology, Faculty of Medicine, University of Alexandria, Alexandria, Egypt.

\section{Funding}

The authors report no funding for this study.

\section{Disclosure}

The authors declare that there is no conflict of interest.

\section{References}

1. Adamopoulou C, Rao RC. Surgical correction of consecutive esotropia with unilateral medial rectus recession. J Pediatr Ophthalmol Strabismus. 2015;52(6):343-347. doi:10.3928/01913913-20150929-16

2. Wang L, Nelson LB. Outcome study of unilateral lateral rectus recession for small to moderate angle intermittent exotropia in children. J Pediatr Ophthalmol Strabismus. 2010;47(4):242-247. doi:10.3928/ 01913913-20091019-12

3. Gurland J, Vagge A, Nelson LB. One-muscle strabismus surgery: a review. J Pediatr Ophthalmol Strabismus. 2018;55(5):288-292. doi:10.3928/01913913-20180327-03
4. Scott AB Graded rectus muscle tenotomy for small deviations. Proceedings of the Jampolsky Festschrift. San Francisco: The SmithKettlewell Eye Research Institute; 2000:215-216.

5. Yim HB, Biglan AW, Cronin TH. Graded partial tenotomy of vertical rectus muscles for treatment of hypertropia. Trans Am Ophthalmol Soc. 2004;102:169-176.

6. Archer SM. Small deviations: vertical, horizontal, and combined. Am Orthopt J. 2015;65:31-34. doi:10.3368/aoj.65.1.31

7. Yau GSK, Tam VTY, Lee JWY, Chan TTK, Yuen CYF. Surgical outcomes for unilateral superior oblique palsy in Chinese population: a retrospective study. Int $J$ Ophthalmol. 2015;8:107-112. doi:10.3980/j.issn.2222-3959.2015.01.20

8. Hatz KB, Brodsky MC, Killer HE. When is isolated inferior oblique muscle surgery an appropriate treatment for superior oblique palsy? Eur J Ophthalmol. 2006;16(1):10-16. doi:10.1177/112067210 601600103

9. Akbari MR, Sadrkhanlou S, Mirmohammadsadeghi A. Surgical outcome of single inferior oblique myectomy in small and large hypertropia of unilateral superior oblique palsy. $J$ Pediatr Ophthalmol Strabismus. 2019;56(1):23-27. doi:10.3928/ 01913913-20180925-03

10. Ward JB, Niffenegger AS, Lavin CW, et al. The use of propofol and mivacurium anesthetic technique for the immediate postoperative adjustment of sutures in strabismus surgery. Ophthalmology. 1995;102(1):122-128. doi:10.1016/S0161-6420(95)31070-6

11. Zhang MS, Hutchinson AK, Drack AV, Cleveland J, Lambert SR. Improved ocular alignment with adjustable sutures in adults undergoing strabismus surgery. Ophthalmology. 2012;119(2):396-402. doi:10.1016/j.ophtha.2011.07.044

12. Tripathi A, Haslett R, Marsh IB. Strabismus surgery: adjustable sutures-good for all? Eye. 2003;17(6):739-742. doi:10.1038/sj. eye. 6700465

13. Eino D, Kraft SP. Postoperative drifts after adjustable-suture strabismus surgery. Can J Ophthalmol. 1997;32(3):163-169.

14. Gawęcki M. Adjustable versus nonadjustable sutures in strabismus surgery-who benefits the most? J Clin Med. 2020;9(2):292. doi: $10.3390 / \mathrm{jcm} 9020292$

15. Leffler CT, Vaziri K, Cavuoto KM, et al. Strabismus surgery reoperation rates with adjustable and conventional sutures. $\mathrm{Am}$ J Ophthalmol. 2015;160(2):385-390.e384. doi:10.1016/j.ajo.2015. 05.014

16. Tatham A, Amaya L. Immediate post-operative adjustable suture strabismus surgery using a target-controlled infusion of propofol-remifentanil. Int $J$ Ophthalmol. 2009;223(3):192-195. doi:10.1159/000200766

17. Millar JM, Jewkes CF. Recovery and morbidity after daycase anaesthesia. A comparison of propofol with thiopentone-enflurane with and without alfentanil. Anaesthesia. 1988;43(9):738-743. doi:10.1111/j.1365-2044.1988.tb05743.x

18. Biglan AW, Davis JS, Day R, Landsittel D. Prospective investigation of the effectiveness of intraoperative adjustable sutures for correction of strabismus. Trans Am Ophthalmol Soc. 1994;92:325-347.

19. Cogen MS, Guthrie ME, Vinik HR. The immediate postoperative adjustment of sutures in strabismus surgery with comaintenance of anesthesia using propofol and midazolam. J AAPOS. 2002;6 (4):241-245. doi:10.1067/mpa.2002.123398

20. Sharma P, Julka A, Gadia R, Chhabra A, Dehran M. Evaluation of single-stage adjustable strabismus surgery under conscious sedation. Indian J Ophthalmol. 2009;57(2):121-125. doi:10.4103/03014738.45501

21. Bleik JH, Karam VY. Comparison of the immediate with the 24-hour postoperative prism and cover measurements in adjustable muscle surgery: is immediate postoperative adjustment reliable? $J$ AAPOS. 2004;8(6):528-533. doi:10.1016/j.jaapos.2004.08.004 


\section{Publish your work in this journal}

Clinical Ophthalmology is an international, peer-reviewed journal covering all subspecialties within ophthalmology. Key topics include: Optometry; Visual science; Pharmacology and drug therapy in eye diseases; Basic Sciences; Primary and Secondary eye care; Patient Safety and Quality of Care Improvements. This journal is indexed on PubMed

Submit your manuscript here: https://www.dovepress.com/clinical-ophthalmology-journal
Central and CAS, and is the official journal of The Society of Clinical Ophthalmology (SCO). The manuscript management system is completely online and includes a very quick and fair peer-review system, which is all easy to use. Visit http://www.dovepress.com/ testimonials.php to read real quotes from published authors. 